\title{
Philosophiques
}

\section{Quelques remarques sur le jugement}

\section{Josette Lanteigne}

Volume 19, numéro 1, printemps 1992

URI : https://id.erudit.org/iderudit/027170ar

DOI : https://doi.org/10.7202/027170ar

Aller au sommaire du numéro

Éditeur(s)

Société de philosophie du Québec

ISSN

0316-2923 (imprimé)

1492-1391 (numérique)

Découvrir la revue

Citer cet article

Lanteigne, J. (1992). Quelques remarques sur le jugement. Philosophiques, 19(1), 25-43. https://doi.org/10.7202/027170ar

\section{Résumé de l'article}

Le but de ces quelques remarques sur le jugement est de faire apparaître cette notion sous différents éclairages (logique, pragmatique, transcendantal), tout en assurant une certaine progression d'un point de vue à l'autre. Dans la première section, il sera question non seulement de la synthèse au sens kantien du jugement synthétique fondé sur l'intuition, mais également des trois synthèses predicative, véritative et apophantique distinguées par Heidegger et reprises par Lotz. La deuxième section, plus proche de la pensée de Wittgenstein, s'interroge sur le jugement dans le cadre de la " grammaire philosophique ». Enfin, la troisième section examine l'idée d'un primat du jugement sur la base du primat de la raison théorique soutenu par Jacques Poulain. En apparence, cette thèse prend le contre-pied de celle de Kant dans la Critique de la raison pratique, sans qu'on puisse lui attribuer la volonté de fonder une nouvelle " philosophie théorique ".
Ce document est protégé par la loi sur le droit d'auteur. L'utilisation des services d'Érudit (y compris la reproduction) est assujettie à sa politique d'utilisation que vous pouvez consulter en ligne.

https://apropos.erudit.org/fr/usagers/politique-dutilisation/ 


\title{
QUELQUES REMARQUES SUR LE JUGEMENT
}

\author{
par Josette Lanteigne
}

Résumé. Le but de ces quelques remarques sur le jugement est de faire apparaître cette notion sous différents éclairages (logique, pragmatique, transcendantal), tout en assurant une certaine progression d'un point de vue à l'autre. Dans la première section, il sera question non seulement de la synthèse au sens kantien du jugement synthétique fondé sur l'intuition, mais également des trois synthèses prédicative, véritative et apophantique distinguées par Heidegger et reprises par Lotz. La deuxième section, plus proche de la pensée de Wittgenstein, s'interroge sur le jugement dans le cadre de la "grammaire philosophique». Enfin, la troisième section examine l'idée d'un primat du jugement sur la base du primat de la raison théorique soutenu par Jacques Poulain. En apparence, cette thèse prend le contre-pied de celle de Kant dans la Critique de la raison pratique, sans qu'on puisse lui attribuer la volonté de fonder une nouvelle "philosophie théorique".

ABSTRACT. The aim of the following remarks on judgment is to present this notion from different points of view (logical, pragmatical and transcendantal), with a certain progression from the first to the others point of view. In the first section, it will be question not only of synthesis in the kantian sense which bears upon intuition, but equally of the three syntheses (predicative, veritative and apophantic) distinguished by Heidegger and Lotz. The second section, more wittgensteinian in spirit, asks the question of judgment in the context of the " philosophical grammar ». Finally, the third section investigates Jacques Poulain's idea of a primacy of judgment on the basis of the primacy of theoretical reason over practical reason. At first sight, this thesis of Poulain seems to go against Kant's thesis in the Critic of practical Reason, but we don't have to attribute to him the will to establish a new "theoretical philosophy". 
Même s'il ne communique rien, le discours représente l'existence de la communication; même s'il nie l'évidence, il affirme que la parole constitue la vérité; même s'il est destiné à tromper, il spécule sur la foi dans le témoignage ${ }^{1}$.

Ces quelques remarques sur le jugement pourraient être comprises comme un libre commentaire de cette citation de Lacan, même si elle a été trouvée après coup. Disons plutôt qu'elle exprime bien ce que Poulain et plusieurs autres, si on observe bien, pensent et " pensent vrai ". Et je me suis donnée pour tâche d'apporter mon propre jugement à cette pensée, ce qui revient essentiellement à trouver des matériaux auxquels elle se laisse appliquer. L'intervention de Kant ne causera pas de surprise, puisque Poulain se réfère explicitement à lui et aux jugements synthétiques a priori. Quant à Wittgenstein, il intervient plutôt comme figure sujette à la critique, au bon sens kantien du terme "critique", ce qui permet à la pensée de Wittgenstein de rester la toile de fond sur laquelle se déroule ce jeu de langage, alors que Poulain fournit l'inspiration et Kant l'orientation sur le jugement.

\section{Le jugement comporte une synthèse et même plusieurs}

En distinguant les jugements analytiques des jugements synthétiques dans sa première Critique, Kant ne dissimule pas que sa préférence va aux seconds, qui constituent à ses yeux les seuls jugements susceptibles de faire progresser la connaissance, et qu'il appelle pour cette raison "jugements extensifs". Les jugements synthétiques se distinguent des jugements analytiques en ce qu'ils comportent non seulement une analyse de concepts, mais également une synthèse des représentations. Or suivant Kant, ce terme de "synthèse " présuppose un "substrat " qui sera l'expérience pour les jugements empiriques, mais qui devra être l'intuition pour les jugements synthétiques en général (a priori ou a posteriori). Qu'appelle-t-on «intuition »? D'entrée de jeu, l'intuition kantienne a toujours pour but la "synthèse des phénomènes " (ou l'expérience, suivant l'acception kantienne du terme), indépendamment de toute vision intellectuelle des choses en soi. Toutefois, l'intuition sensible et finie, étant aveugle par elle-même, a besoin d'être soumise à l'éclairage du concept. Et comme celui-ci est de son côté une simple forme vide de pensée, on doit lui apporter l'intuition qui lui est nécessaire pour recevoir un contenu, ce qui ne peut se faire que par un 
acte de la pure spontanéité de l'esprit. Cet acte est ce que Kant appelle jugement:

Je n'ai jamais pu me satisfaire de la définition que donnent les logiciens d'un jugement en général: c'est, à ce qu'ils disent, la représentation d'un rapport entre deux concepts [...]

Mais si je recherche plus exactement la relation des connaissances données dans chaque jugement, et si je la distingue, comme appartenant à l'entendement, du rapport établi d'après les lois de l'imagination reproductrice (rapport qui n'a qu'une valeur subjective), je trouve qu'un jugement n'est rien d'autre que la manière d'amener des connaissances données à l'unité objective de l'aperception ${ }^{2}$.

La première portion de la citation pourrait convenir comme description des jugements analytiques (définis dans la section IV de l'introduction à la Critique de la raison pure comme jugements dont le prédicat est implicitement contenu dans le concept du sujet), alors que la deuxième proposerait la seule véritable définition des jugements synthétiques a priori (présentés au début de la Critique comme un "mystère »). En d'autres termes, la " nouvelle délimitation de l'essence du jugement " ${ }^{3}$ proposée par Kant serait celle du jugement synthétique a priori. Comme on l'a dit plus haut, Kant ramène tous les jugements synthétiques à l'intuition, mais il faut remarquer aussi que ce n'est pas de la même manière, puisque les jugements synthétiques a posteriori s'appuient sur l'intuition empirique, alors que les jugements synthétiques a priori la précèdent en produisant ce que Kant appelle l'« unité de l'intuition » à travers les catégories. La sorte de jugements synthétiques que Kant valorise le plus ne regarde pas directement vers l'intuition mais depuis-leconcept-vers-l'intuition, ce qui fait que même si l'intuition a un rôle central dans la Critique, ce n'est pas elle qui a la première place. De même que la catégorie ne s'applique pas directement à l'objet (celui de l'intuition empirique) mais uniquement à son "schème", le jugement synthétique a priori n'est pas directement fondé sur l'intuition (à l'exception des jugements de la mathématique, dont les "constructions"s'élaborent en s'appuyant sur l'intuition a priori), mais sur la synthèse de l'intuition opérée par l'imagination et l'entendement.

S'agissant du concept, le maître mot est l'intuition, mais s'agissant du jugement, il est plutôt synthèse. Celle-ci implique d'abord deux termes, puis trois: ainsi, Kant définit la connaissance comme la synthèse de l'intuition et du concept $t^{4}$, mais la " connaissance " est elle-même un troisième terme, dont on 
ne peut faire abstraction sitôt qu'on délaisse le champ des connaissances empiriques, où le sujet ne distingue pas la perception de ses paroles de la perception de l'objet. Kant distingue le jugement objectif de connaissance: "la pierre est lourde ", du simple jugement de perception: «il me semble que la pierre est lourde ${ }^{5}$. La liaison des représentations est empirique dans les deux cas, mais elle s'accompagne dans le jugement objectif d'une conscience de sa nécessité qui ne saurait lui venir que du sujet qui juge en fonction de ce qu'il reconnaît ne pas pouvoir ne pas penser vrai. Dans ce contexte, Kant est très attentif à dissocier la véritable connaissance, qui est toujours une connaissance synthétique a priori qui anticipe sa propre vérité transcendantale, des associations libres de l'imagination reproductive, qui n'ont qu'une valeur subjective. Mais sur quoi Kant pourrait-il asseoir la supériorité des lois de l'entendement sur celles de l'imagination, si ce n'est justement sur le mouvement par lequel nous reconnaissons comme objectives uniquement des pensées auxquelles nous avons dû adhérer pour pouvoir les penser vraies, ce qui démontre sans doute notre engagement profond à l'endroit des notions de vérité et d'objectivité, mais sans nous libérer de l'identification aux sons rendant possible toute réalité humaine ${ }^{6}$.

Herder a été le premier d'une longue lignée de penseurs à reprocher à Kant d'avoir été aveugle à la dimension linguistique. Et tout indique que si Kant avait tenu compte du langage, il aurait dû le faire dans le cadre du schématisme. Kant attribue un schématisme à l'imagination transcendantale, mais n'auraitil pas avec autant de pertinence pu l'attribuer au langage? La théorie du tableau de Wittgenstein n'est-elle pas manifestement un schématisme propositionnel? Kant n'aurait pas eu besoin de décrire le schématisme comme "un art caché dans les profondeurs de l'âme humaine ${ }^{7}, \mathrm{~s}^{\prime} \mathrm{il}$ l'avait attribué au langage, puisque le langage est public par définition. Ce que Kant appelle «synthèse », d'autres pourraient l'appeler "discursivité », un terme qui s'apparente aussi bien à la nécessité pour l'intuition finie d'être médiatisée par le concept, qu'il ne s'apparente au "discours" et donc au langage.

Heidegger distingue trois synthèses: prédicative, véritative et apophantique. La synthèse prédicative consiste à penser un concept comme relié à un autre au moyen de la copule; cette synthèse présuppose la synthèse véritative, qui ordonne tout le contenu du jugement à l'objet et la synthèse apophantique, qui est l'acte de poser l'être ${ }^{8}$. On peut rapprocher ces différentes 
synthèses, respectivement, du jugement analytique (synthèse prédicative des concepts dans un jugement), du jugement synthétique (synthèse véritative du concept et de l'intuition dans un jugement de perception) et du jugement synthétique a priori (synthèse apophantique de l'unité objective de l'aperception dans un jugement transcendantal). Mais quels sont, indépendamment de Kant, les rapports entre ces différentes synthèses? La nécessité de dépasser la définition traditionnelle du jugement comme synthèse prédicative du concept du sujet avec le concept du prédicat semble acquise. La lutte se fait donc entre synthèse véritative et synthèse apophantique. Toutefois, Heidegger serait le premier à reconnaître qu'il s'agit là d'un faux débat, puisque la synthèse de l'étant (synthèse véritative) et celle de l'être de l'étant (synthèse apophantique) ne sauraient être dissociées l'une de l'autre. Suivant l'exemple de Apel, qui tire lui-même son modèle de Peirce', on peut tracer un tableau des théories qui surgissent lorsqu'on accorde la priorité à l'une ou à l'autre des différentes synthèses prédicative (I), véritative (II) ou apophantique (III):

I sans II et III: "Tous les jugements sont analytiques, ou alors je ne sais pas ce qu'est la vérité " (Leibniz repris par Wolf).

II dominant I et III: La synthèse véritative englobe les deux autres (position de J. B. Lotz et de Heidegger).

III sans I et II: Métaphysique de l'être.

I: Que le jugement comporte non seulement une analyse de ses différents concepts mais également une synthèse, un tout, c'est ce que personne ne songerait à nier. En effet, il serait absurde de considérer que tous les jugements sont analytiques: " $\mathrm{Si}$ je dis: "mon balai se trouve dans le coin" - s'agit-il vraiment d'un énoncé concernant le manche à balai et la brosse du balai? " ${ }^{10}$. Les jugements sont "analysés " une fois qu'on les a tirés de leur contexte; qu'on puisse se livrer à cet exercice montre peut-être quelque chose (à savoir, que la logique est l'essence du langage ${ }^{11}$, mais on ne peut pas le dire), mais ne dit pas que tous les jugements sont analytiques.

II: Selon J. B. Lotz, "le jugement dit ce que l'individu est ${ }^{12}$. A-t-on affaire ici à la synthèse prédicative (où quelque 
chose est prédiqué du sujet), à la synthèse véritative (qui ordonne tout le contenu du jugement à l'objet), ou à la synthèse apophantique (l'acte de poser l'être)? De toute évidence, Lotz soumet le jugement à l'autorité de la synthèse véritative, comme Kant et comme Heidegger. Il préférerait même éviter de parler de synthèse apophantique, "parce que la vérité, et donc aussi la synthèse véritative, englobent expressément deux aspects, celui de la relation à l'objet et celui de l'acte de position, tandis qu'on peut encore faire abstraction de l'acte de position quand il s'agit de la synthèse prédicative $~_{13}$. La synthèse prédicative et la synthèse apophantique présupposeraient donc toutes deux la synthèse véritative, ce qui expliquerait qu'elles soient condamnées à l'échec lorsqu'on cherche à imposer de manière exclusive le point de vue de la proposition (der Satz) ou celui de l'Être. Quant à la synthèse véritative, sans avoir besoin d'attendre les résultats de l'analyse propositionnelle, elle englobe la synthèse apophantique. Si je dis "mon balai se trouve dans le coin", je ne fais pas un énoncé sur la brosse et le manche à balai, mais je le rends disponible pour un futur usage, j'anticipe son utilisation comme balai (muni d'une brosse et d'un manche à balai mais de cette analyse, je fais complètement abstraction lorsque j'anticipe son utilisation).

III: Pour Lotz, l'acte de poser est le principe suprême; les phases antérieures sont le poser en route vers lui-même, la marche de l'être en route vers lui-même. Lotz n'est satisfait que lorsque le jugement devient l'être. Mais le jugement $\mathrm{n}^{\prime}$ est-il pas alors en danger de s'oublier lui-même au profit de l'être? L'ambiguité est présente chez Heidegger aussi bien que chez Lotz; car dans les deux cas, on assiste à l'irruption d'une métaphysique de l'être qui soumet la synthèse véritative à ses fins. Cette problématique est étrangère à Kant, dont le problème est plutôt de dépasser la synthèse prédicative vers la synthèse véritative, mais on la remarque chez ses successeurs immédiats, qui réintroduisent une forme de métaphysique en dépit des critiques de Kant, comme si la chose pouvait encore avoir un sens, pour un "esprit absolu " ou un "génie".

\section{Le jugement comme jeu de langage}

Wittgenstein fut un des premiers avec Peirce à avoir dénoncé avec autant de vigueur les fixations mentales ("significations", "processus vécus », " actes mentaux», etc.) censées accompagner 
l'énonciation des propositions. Il affirme volontiers: «Ne vous demandez pas ce que vous pensez, mais ce que les autres pensent ». La détermination wittgensteinienne de l'essence du jugement diffère de celle de Kant par l'importance accrue qu'il accorde au facteur transsubjectif: "Si le langage doit être un moyen de communication, il doit y avoir un accord, non seulement au niveau des définitions mais aussi (si étrange que cela puisse paraître), dans les jugements " ( $P U, 242)$. De toute évidence, le jugement n'est pas d'abord et avant tout pour Wittgenstein une connaissance objective mais une réalité intersubjective. Ainsi, au lieu de dire que si l'incompréhension est le lot quotidien de ceux qui parlent la même langue, c'est parce qu'ils donnent aux mots des sens différents, on peut remarquer avec Wittgenstein que même si nous définissions nos termes de façon identique, les jugements qu'on sera amené à faire pourraient bien différer par la suite. On ne saurait anticiper le jeu de langage du jugement au moyen des définitions, car le " jeu de langage" est imprévisible ${ }^{14}$. Faudrait-il tenter de définir ce vocable de Wittgenstein? À moins qu'il ne faille le considérer comme un jugement? Mais un jugement ne requiertil pas un verbe? Les "verbes" se conjuguent avec l' «action", et Wittgenstein définit le jeu de langage comme les mots plus leur intrication dans nos actions $(P U, 7)$, plus leurs connexions entre eux, puisqu'il est clair que cette "connexité grammaticale " des mots tient lieu de correspondance à la réalité, en un sens paradigmatique et non métaphysique ( $P U, 131$ ).

Ce qui distingue le jeu de langage d'une simple compréhension du sens des mots, c'est la prise en considération de l'existence des locuteurs et de leurs actions. Les actes de langage étudiés par Wittgenstein sont des plus "ordinaires": celui de continuer une série en mathématiques, par exemple, plutôt que la série en elle-même, ses lois et sa pertinence. Malgré tout, ses remarques sur les mathématiques sont parfois difficiles à distinguer de celles que ferait un mathématicien. On sait que Wittgenstein revendiquait pour les mathématiques le fait d'être conceptuelles. Mais le concept wittgensteinien est assez éloigné du concept au sens kantien: dépourvu de signification abstraite, il n'a pas besoin d'être relié à l'objet de l'intuition sensible, sinon dans un sens inverse de celui de Kant. En d'autres termes, si Wittgenstein II s'intéresse tant à l'usage des "concepts", c'est qu'il ne s'intéresse plus guère aux "objets " ${ }^{15}$. Les règles auxquelles obéissent les concepts sont uniquement grammaticales. Nous essaierons de le rendre manifeste de la manière la plus concrète possible. 
Les remarques grammaticales de Wittgenstein ne se laissent assimiler, ni aux jugements analytiques, ni aux jugements synthétiques. Premièrement, même s'ils portent sur des concepts, comme les jugements analytiques, ce que les énoncés de grammaire " analysent " $\mathrm{n}^{\prime}$ est pas la signification des termes mais leur usage ( le balai est dans le coin " ne s'analyse pas en termes de brosse et de manche à balai mais on demande par exemple: "Qu'appelle-t-on "un balai"? »16; "Comment employons-nous cette expression dans nos jeux de langage? ${ }^{17}$ ). Deuxièmement, contrairement aux jugements synthétiques cette fois, les remarques grammaticales ne cherchent pas à passer du côté de l'expérience (auquel cas on chercherait à passer de l'expression «balai " à l'expression "dans le coin », où se trouve le balai), mais seulement à décrire des usages multiples et leur connexité ${ }^{18}$, sans se donner d'autres buts qu'une plus grande clarté et que le traitement des problèmes engendrés dans la recherche et l'action par des usages abusifs du langage, et dont on ne peut se guérir autrement qu'en adoptant d'autres usages.

Troisièmement, les remarques grammaticales de Wittgenstein ne sauraient être classées comme jugements synthétiques a priori, ceux-ci étant explicitement bannis dans Wittgenstein und der Wiener $\mathrm{Kreis}^{19}$. Il y a cependant une distinction analogique qu'on peut se permettre de faire: comme les jugements synthétiques a priori, les remarques grammaticales sont à dissocier des simples jugements de perception ou plutôt, dans les cas qui ont occupé le plus souvent Wittgenstein, des jugements $\mathrm{d}^{\prime}$ introspection ${ }^{20}$. On trouve dans la philosophie des mathématiques de Wittgenstein de nombreux exemples de son anti-réalisme et de son anti-psychologisme, et ses remarques sur la philosophie de la psychologie expriment exactement le même genre de critique $^{21}$. Une remarque grammaticale, qu'elle porte sur l'usage d'un concept mathématique ou psychologique, ne doit pas exprimer ce qu'on pense, quand on fait des mathématiques, ou ce qu'on éprouve, lorsqu'on est dans l'attente d'un événement; elle cherche au contraire à nous détourner de ces préoccupations découlant d'illusions et de sublimations grammaticales.

Dans ce contexte, il est plus aisé de réunir les interprètes de Wittgenstein contre les vieux démons qu'il exorcise que de déterminer ce qu'il cherchait à promouvoir. Le jugement applique-t-il une règle dans l'esprit de l'interprétation communautaire, qui soumet le jugement de chacun à celui de tous ${ }^{22}$ ? Même en accordant son point à l'interprétation communautaire, il reste que ce n'est pas là tout ce qu'on appelle « jugement »: 
La psychologie du jugement. Car le jugement a aussi sa psychologie.

Il est important qu' on puisse s'imaginer chaque jugement commençant par " je »: " je juge que... "

Chaque jugement porte-t-il donc sur celui qui juge? Non pas, dans la mesure où je ne veux pas que les principales conséquences qui sont tirées le soient en ce qui me concerne mais en ce qui concerne l'objet du jugement. Quand je dis qu' «il pleut", je ne désire pas en général qu'on me réponde: "c'est donc ainsi qu'il te semble ». "Nous parlons du temps, pourrais-je rétorquer, et non de moi ${ }^{23}$.

À la psychologie du jugement, le pragmatisme de l'interprétation communautaire ne fait que substituer une sociologie du jugement. Ni l'une ni l'autre ne répondent à la question de savoir en quoi consiste ce qu'on appelle "objectivité du jugement". Même s'il n'est pas assuré qu'un Wittgenstein aurait pu voir là autre chose qu'un faux problème, la psychologie et la sociologie du jugement sont certainement de fausses solutions: Même si on peut s'imaginer que tous les jugements commencent par «je juge que...» (Kant affirme bien que le «je pense» doit pouvoir accompagner toutes mes représentations $\left.{ }^{24}\right)$, cela ne fait pas d'un sujet quelconque qui pense l'objet du jugement.

Même si tous les jugements doivent pouvoir être communiqués, on ne peut dire que la communication soit la racine du jugement, car la possibilité de communiquer dans le langage doit déjà exister pour qu'on puisse " parler" de " communication". Le fait que l'" accord " soit un ingrédient essentiel du jugement et du jeu de langage (en admettant qu'il le soit), n'implique aucunement que le jeu de langage du jugement soit fondé sur un accord (agreement, Übereinstimmung); le fait que le consensus soit nécessaire (et aussi arbitraire que nécessaire), empêche de s'en prévaloir comme d'une base objective. Tout l'aspect "humain" du consensus se trouve ainsi dissout par la grammaire: la notion de "consensus " est soumise à des règles grammaticales que nous n'avons pas parce qu'elles nous permettent de communiquer, même si nous pouvons trouver pratique de communiquer au moyen de telles règles.

\section{Le primat du jugement}

On peut tenter d'éclairer la question du jugement en s'appuyant sur les trois maximes kantiennes du sens commun: I) penser par soi-même; il) mettre son jugement en accord avec celui des autres; II) penser de manière conséquente ${ }^{25}$. On peut attribuer à Kant la première position dans la Critique de la raison pure, 
où le jugement est décrit comme un acte qui relève essentiellement de l'entendement. D'autres "facultés » président au jugement en plus de l'entendement, mais c'est lui qui est défini comme le "pouvoir des règles". La nouvelle définition wittgensteinienne du jugement est pratiquement identique à la deuxième maxime. Quant à la troisième maxime, la plus difficile à suivre selon Kant, elle ne désigne pas le principe logique de non-contradiction ${ }^{26}$, mais la nécessité de penser son jugement en accord avec soi-même, c'est-à-dire de reconnaître sa vérité, pour pouvoir le penser. En résumé, Kant découvre la nécessité de penser par soi-même, Wittgenstein celle de mettre son jugement en accord avec celui des autres, la deuxième condition renvoyant à la première, sans qu'on réalise que cet accord avec soi est une identification primitive à la vérité qui précède et conditionne tout accord de mon jugement (Kant) avec celui des autres (Wittgenstein).

On doit attribuer la maxime du penser vrai à Jacques Poulain ${ }^{27}$. Il $s^{\prime}$ agit en fait $d^{\prime} u n e$ nouvelle synthèse véritative. La manière dont cette synthèse ordonne tout le contenu du jugement à l'objet consiste à reconnaître l'absence de différence entre la proposition qui est objectivement vraie et la réalité décrite par elle, qui reproduit l'identité primitive du son émis et du son reçu, dans l'émission-réception phono-auditive. Sans entrer dans les détails de la formation du langage comme système autonome (Poulain s'appuie sur Gehlen, Tomatis, etc.), que l'individu peut utiliser comme "outil de communication", par exemple, je tenterai d'appliquer cette identification primitive au jugement, ou plutôt de reconnaître en lui une identification primitive au vrai, c'est-à-dire un jugement de vérité.

Un premier stade du jugement est celui de la proposition: «le soleil brille». Ici, celui qui juge ne distingue pas ses propres paroles du fait qu'elles décrivent, pas plus qu'il ne distingue le soleil de sa propriété de brillance. Un deuxième stade est celui de la proposition sujet-prédicat. Dans «le soleil est brillant », la propriété de brillance est nettement différenciée par rapport à l'objet, mais c'est uniquement parce qu'on a substitué au rapport à l'objet un autre rapport: au lieu de déclencher la perception de la propriété de brillance, "soleil " comme sujet déclenche maintenant l'action d'énoncer le prédicat " est brillant ${ }^{28}$. Ni l'un ni l'autre des deux premiers stades ne mérite d'être appelé "jugement», car il leur manque encore la conscience de vérité. Au premier stade, celui de la "prosopopée 
verbale ", il n'est pas encore question de vérité29; et au second stade de la proposition sujet-prédicat (synthèse prédicative), il ne saurait non plus en être question, si la relation $S / P$ est fondée sur la déconnexion du sujet d'avec le référent et sur celle du prédicat d'avec la propriété qu'on se faisait percevoir au premier stade.

Le troisième stade du jugement de vérité est celui où on doit penser vrai ce qu'on dit et pouvoir le dire. La première partie de la phrase précédente est une métaphore du système auditif (réceptif), où la parole est en quelque sorte inhibée (en dépit du fait que la parole est rendue possible par l'écoute); la seconde partie évoque l'inlassable activité motrice de la parole. Le schéma conventionnel "émission-réception-phase consommatoire» est ici perturbé de la manière suivante: ce qui relève de la pensée comme réceptivité ( $s$ 'imaginer, anticiper, penser, évoquer, etc.) est vécu sur le mode volitif, dans la mesure où le discours, qui conserve toute sa force illocutoire, est l'objet d'une phase consommatoire qui renforce le désir de vérité. Dans « il est aussi vrai que le soleil brille au moment où j'écris ceci qu'il est vrai que je dois le penser vrai pour pouvoir le dire", on trouve plus qu'une volonté de décrire la réalité, une volonté de dire la vérité.

Qu'est-ce que la vérité? C'est par cette question, dit Kant, que les anciens tentaient de pousser les logiciens dans leurs derniers retranchements. Le diallèle auquel ils se trouvaient alors acculés est décrit par les traducteurs de la première Critique de la manière suivante: "Il y a diallèle parce que l'esprit prétend se laisser mesurer par le réel, mais établit lui-même cette mesure" ${ }^{30}$. On sait que Kant "accorde" et "présuppose » la définition nominale de la vérité comme conformité de la connaissance avec son objet ${ }^{31}$. Mais son affirmation peut surprendre dans le contexte de l'idéalisme transcendantal L'idéalisme kantien ne cherche-t-il pas à valider un diallèle? On retrouve le même genre d'inversion dans les articles de Poulain: l'objectivité et la vérité y sont présupposées tout en recevant une nouvelle détermination qui doit précisément "déterminer " ce qui était présupposé.

Nous avons un préjugé favorable à l'endroit du jugement. Comme le remarque Wittgenstein, "nous utilisons des jugements comme principes de l'acte de juger ${ }^{32}$. De prime abord, cette folie du jugement ne présente qu'une autre forme d'animisme. $\mathrm{Ne} s^{\prime} y$ limite-t-on pas à la seule expérience de vérité que 
l'on s'est fait faire, celle d'une vérité nécessaire (le faux ne se laissant pas "penser" de cette manière), en faisant fi de la polarité essentielle à ce concept? Comme dit Wittgenstein dans le Tractatus, la proposition vraie présuppose la proposition négative et vice versa ${ }^{33}$. Toutefois, ce qui s'oppose au jugement de vérité n'est pas la proposition négative mais celle qui est fausse. Et il serait absurde de croire que le faux doive logiquement se laisser penser en même temps que ce qui est vrai. L'obligation est plutôt de penser vrai ce qui est affirmé et de déterminer comme faux ce qu'on ne peut penser vrai. Mais Poulain va plus loin lorsqu'il affirme que le jugement "fixe à lui-même et à l'expérience qu'il objective si et seulement s'il est aussi vrai qu'il a dû se penser l'être pour pouvoir se produire ${ }^{34}$. Il fait ainsi de tout jugement un jugement synthétique a priori, comme Kant définissait tout jugement véritable comme un jugement synthétique a priori au $\S 19$ de la deuxième édition de la Logique transcendantale. Dès lors, "penser vrai " seraitil "juger" au sens kantien du terme? Le cas échéant, on pourrait lui opposer l'argument présumé valable contre toute philosophie de la conscience, à savoir que l'unité de l'aperception doit céder à l'unité du consensus locuteur/allocutaire, si le jugement doit pouvoir être non seulement pensé mais communiqué.

Suivant la pragmatique transcendantale, l'unité objective de l'aperception serait une invention de philosophe contemplatif, pour qui l'objectivité consiste à passer de «il me semble que la pierre est lourde » à «la pierre est lourde » (= «il en est ainsi »). Si "il en est ainsi " doit suffire dans la situation du philosophe des sciences de la nature, le philosophe des sciences de l'esprit ne se tient pour assuré de rien qui n'ait été soumis à l'épreuve du discours argumentatif. En définissant le jugement en termes d'accord, le pragmatisme est uniquement conséquent, comme le scientisme l'était en le définissant en termes d'objectivité nécessaire. Ils sont conséquents, mais ils ne pensent pas de manière conséquente (troisième maxime du jugement et troisième stade du jugement), en mettant leur jugement en accord avec ce qu'ils disent. Le jugement scientifique doit se présumer aussi objectif qu'il peut démontrer qu'il l'est: sa démonstration ne souscrit donc pas aux critères d'une démonstration scientifique indépendante; le pragmaticien pense déjà vraies les conditions idéales qu'il cherche à réaliser: comment le consensus seraitil indisponible, puisqu'on le cherche au lieu de reconnaitre qu'on l'a déjà présupposé ${ }^{35}$ ? La métaphysique pragmatiste traite la pensée comme un désir. Or ce qui distingue le jugement 
d'un désir est qu'on s'y identifie à une réalité en reconnaissant la vérité de ce qui est pensé, tout en refusant de sortir du langage pour reconnaître un autre rapport à la réalité que celui-là.

Au primat de la raison pratique, institué par Kant et jamais remis en question par Apel et par Habermas, Poulain propose de "substituer celui de la raison théorique, et ce dans le domaine même de la raison pratique, au sein des rapports éthico-politiques ${ }^{36}$. Pourtant, Poulain n'a pas de "philosophie théorique " à proposer, de toute évidence. De là à soutenir qu'il a au fond une philosophie pratique, il n'y a qu'un pas. Ne considère-t-il pas le jugement comme une expérience? Mais l'expérience en question en est une d' "auto-objectivation", le "mouvement théorique" par lequel on se fait reconnaître la vérité de ce qu'on s'est fait penser. Justement, où est la différence entre "se faire reconnaître" et "se faire penser"? Ne s'agit-il pas à chaque fois d'un "se faire faire " quelque chose? On a associé plus haut le travail de Poulain à la synthèse véritative, de façon purement nominative. On peut maintenant préciser que la vérité doit être "pensée vraie", que nous devons (nous) la faire penser vraie. Toutefois, "penser vrai" n'est pas une action comme peut l'être le fait de lever un bras: ce à quoi on se fixe est un certain concept. On retrouve en Poulain un wittgensteinien de l'espèce la plus pure. Comme Wittgenstein, Poulain "délivre de la fascination exercée par l'identification totémiste à la volonté de toute puissance, sans pouvoir prétendre ajouter quelque iota que ce soit à la puissance de juger qu'[il] libère ${ }^{37}$. Poulain ne nous propose pas, en bon chaman, une nouvelle identification pour remplacer les anciennes, mais il nous invite à les reconnaître toutes pour ce qu'elles sont, et à reconnaître dans ce mouvement (ir-)réflexif ce qui nous permet également $d^{\prime} y$ adhérer en toute objectivité.

Poulain serait sans doute le premier à admettre qu'il s'agit là d'un simple jeu de langage. Mais si le jeu de langage du jugement de vérité rend possible le langage et par là, la vie humaine, il est suivant l'expression de Apel un « jeu de langage transcendantal ». À quoi tient le primat du jugement, sinon au fait que ce mouvement théorique d'auto-objectivation est à la source de tout mouvement de communication sensible, de communication par le langage, par la représentation, etc., en tant qu'il s'agit dans tous ces cas de mouvements de réflexion qui se perçoivent comme tels (même si le langage est seul à 
pouvoir le dire) et qui n'ont pas d'autre signification que celle du penser vrai (auquel on n'attribue aucune « force illocutoire " magique), pas d'autre conséquence que celle de nous mettre "en accord avec nous-mêmes ". Au lieu d'être ce qui permet au philosophe de communiquer avec tous les jeux de langage, le jeu de langage transcendantal du jugement anticipe le succès de la forme de vie, succès que celle-ci ne peut que présupposer. Il ne s'agit pas là d'une explication pragmatique, sinon au sens du mouvement réflexif et régulateur par lequel le jugement doit se penser vrai (mouvement théorique) pour pouvoir se produire (pratiquement), étant entendu que «se produire» est $d^{\prime}$ abord et avant tout ce qui doit être pensé vrai.

\section{Conclusion}

Pour Kant, le jugement au sens fort de la Critique de la raison pure $n^{\prime}$ est pas un acte individuel ${ }^{38}$ (tel le jugement de perception) ou particulier (une relation entre deux concepts), puisqu'il le conçoit comme nécessaire et objectif. C'est cette forme de jugement (qui n'est pas la seule) qu'on a cherché à questionner ici. Qu'appelle-t-on «jugement »? Ce que Kant dit, c'est que la synthèse prédicative (ou les « jugements analytiques") ne suffit pas à rendre compte de l'objectivité de la connaissance. Mais ce qu'il démontre, c'est que la subjectivité transcendantale est à la racine de toute objectivité. Même objectif, le jugement reste donc subjectif en un sens supérieur, comme le jugement synthétique a priori ne pouvait être une connaissance rationnelle par concepts seulement, mais devait se rapporter à l'intuition empirique ou à la forme pure de l'intuition sensible, espace et temps. La pensée subséquente a vite fait de remplacer la subjectivité transcendantale par une forme d'intersubjectivité a priori. Finalement, si le langage doit être un moyen de communication, le jugement ne saurait être que le produit de l'adhésion commune des locuteurs à la vérité de ce qu'ils disent. Toutefois, le pragmatisme pur et simple est régressif: de même que l'entendement supérieur de Kant a dû "sensibiliser » la raison, en cédant au pouvoir $\mathrm{du}$ consensus, la pragmatique transcendantale doit " relativiser " le jugement.

Juger est-il encore pour Wittgenstein l'acte de penser par soi-même? Sa "nouvelle définition du jugement" en $P U, 242$ pourrait apporter de l'eau au moulin de l'interprétation communautaire, si Wittgenstein ne précisait déjà sa pensée dans le paragraphe qui précède: ce qu'on trouve dans le jugement 
n'est pas une "conformité d'opinion" mais une "conformité de forme de vie». Si on réduit le jugement à une réalité intersubjective (ce qui n'est pas exactement la conception wittgensteinienne, même si on lui a généreusement attribué cette interprétation), la conception de Jacques Poulain semble plus proche de celle de Kant que de celle de Wittgenstein. Mais Poulain réalise également les promesses de la notion de forme de vie, en prenant le langage pour base réelle du jugement.

$\mathrm{Si}$ on voulait proposer une autre définition du jugement, après celle des logiciens (le jugement est une relation entre deux concepts), après celle de Kant (le jugement est l'acte de ramener les connaissances données à l'unité objective de l'aperception) et après celle de Wittgenstein (le jugement est une forme de vie, l'accord dans le jugement est ce qui permet $q^{\prime}{ }^{\prime} i l$ y ait entente au moyen du langage), on devrait retenir des auteurs précédents quelques termes clefs (la nécessité objective, $l^{\prime}$ « accord dans le jugement $\left.»\right)$, auxquels viendrait s'ajouter le mouvement théorique du "penser vrai ». Le jugement de vérité serait ainsi l'acte de reconnaitre la nécessité et l'objectivité des jugements qu'on a dû penser vrais (dans une identification primitive ayant pour base le consensus) pour pouvoir les penser (ce qui s'appelle "penser vrais » et qui est plus qu'une synthèse aveugle, puisqu'il $s^{\prime}$ agit de l'acte de se fier aux seuls mouvements qui rendent la vie humaine possible).

Docteur en philosophie, Paris VIII Montréal, Canada

\section{NOTES}

1. Jacques Lacan, "Fonction et champ de la parole et du langage en psychanalyse ", dans Écrits I, Paris, Éditions du Seuil, 1966, p. 128.

2. E. Kant, Critique de la raison pure, trad. J.-L. Delamarre et $F$. Marty, dans CEuvres philosophiques, t. I, Paris, Gallimard, 1980 (cette édition donne en marge la pagination de l'édition de l'Académie des sciences de Berlin; abréviation: Ak. III, 114; B 140-141); trad. E. Tremesaygues et B. Pacaud, Paris, P.U.F., 1990 (abréviation: CRP), pp. 118-119.

3. Voir M. Heidegger, Qu'est-ce qu'une chose?, trad. J. Reboul et J. Taminiaux, Paris, Gallimard, 1971, pp. 161, 167.

4. Ak. III, 74; A 50 B 74; CRP, p. 76. 
5. Cf. Ak. III, 113-114; B 140-141; CRP, pp. 118-120.

6. J. Poulain, "Peut-on guérir de la politique? », p. 527. Cf. bibliographie, note 27.

7. Ak. III, 136; A 141 B 180; CRP, p. 153.

8. Cf., M. Heidegger, Kant et le problème de la métaphysique, trad. A. de Waelhens et W. Biemel, Paris, Gallimard, 1953, pp. 89-90 et J. B. Lotz, Le jugement et l'être, trad. R. Givord, Paris, Beauchesne, 1963, pp. 77-80.

9. K. O. Apel, "Zur Idee einer transzendentalen Sprach-Pragmatik ", dans J. Simon, directeur de la publication, Aspekte und Probleme der Sprachphilosophie, Freiburg/München, Verlag Karl Alber, 1974, pp. 286-287.

10. Philosophische Untersuchungen, Oxford, B. Blackwell, 1953 (abréviation:PU), partie I, § 60 .

11. Cf. Tractatus logico-philosophicus (abréviation: T), London, Routledge \& Kegan Paul, 1961, 6.121, 6.124, 6.13.

12. J. B. Lotz, Le jugement et l'être, p. 69.

13. Ibid., p. 81, note 61 .

14. Über Gewissheit, Oxford, B. Blackwell, 1969; De la Certitude, trad. J. Fauve, Paris, Gallimard, 1976, § 559.

15. On sait que dans le Tractatus, "objet " est un concept formel ( $\mathrm{T}$ 4.126). Cf. C. Chauviré, Ludwig Wittgenstein, Paris, Seuil, 1989, p. 86.

16. Contrairement à ce qu'on pourrait croire, le fait de présenter un balai $n^{\prime}$ est pas la meilleure réponse possible à cette question, puisque cette " définition ostensive » fait partie de la grammaire du mot "balai" .

17. Wittgenstein se préoccupe aussi peu des constituants réels du terme " balai » que de l'objet réel qui lui correspond, tandis qu'on observe chez Kant un intérêt marqué pour le "concept réel » de l'objet.

18. Il serait difficile de nier que les expressions "décrire des usages multiples » et "décrire ce qui se passe au sein de l'expérience " appartiennent au même jeu de langage et pourtant, la question est de savoir si la description se fait au moyen de remarques grammaticales ou au moyen de propositions empiriques. On a là un cas où des expressions auxquelles on accorde plus ou moins le même sens peuvent donner lieu à des jugements radicalement opposés.

19. "Anti-Husserl", Wittgenstein und der Wiener Kreis, Notes de F. Waismann sur les conversations de Wittgenstein avec les membres du Cercle de Vienne, Oxford, B. Blackwell, 1967, pp. 67-68. 
20. Wittgenstein souligne qu'il ne rapporte pas ses expériences d'avoir agi suivant son inspiration ou même suivant une règle, lorsqu'il se limite à formuler des "notes grammaticales" ( $P U$ 232).

21. Cf. M. Budd, Wittgenstein's Philosophy of Psychology, London \& New York, Routledge, 1989.

22. Suivant l'interprétation communautaire, la communauté à laquelle nous appartenons fournit la base à partir de laquelle il est possible de justifier une manière de procéder qui ne peut se prévaloir en elle-même d'aucune nécessité objective. Comme dit Kripke, nous suivons la règle aussi longtemps que cette communauté ne nous a pas exclu. (Wittgenstein on Rules and Private Language, Cambridge, Massachusetts, Harvard University Press, 1982, p. 95.)

23. Bemerkungen über die Philosophie der Psychologie, Oxford, B. Blackwell, 1980 , t. I, § 750 .

24. Ak. III, 108; B 131; CRP, p. 110.

25. Voir Ak. V, 294; Critique de la faculté de juger, dans CEuvres philosophiques, t. II, Paris, Gallimard, 1985, § 40.

26. Si c'était le cas, Kant aurait aussi bien pu nommer la maxime de la pensée conséquente en premier, comme il le fait habituellement pour le principe de contradiction, dans sa présentation du principe suprême de tous les jugements analytiques par opposition aux jugements synthétiques a priori (Ak. III, 142; A 150 B 189; CRP, p. 157-158), ou dans son opposition entre la logique générale (qui ne s'intéresse qu'à la forme des connaissances) et la logique transcendantale (où il ne suffit pas que les connaissances ne se contredisent pas pour s'accorder entre elles).

27. Cf. J. Poulain, "Une reconstruction philosophique du concept de Dieu est-elle aujourd'hui possible? », Recherches de science religieuse, t. 64, no 1 (1976), p. 39-58; "Le projet pragmatique : pragmatique de la parole et pragmatique de la vie", Dialogue, vol. XVIII, no 2 (1979), pp. 175-207; “ Vers une pragmatique nucléaire de la communication ", Dialogue, vol. XVIII, no 4 (1979), p. 471-499; "Les paris de Saul Kripke. Une pragmatique rigide du vrai est-elle possible? ", Critique, t. XXXVI, n³99-400, p. 901-911; " Dynamique de la communication et vérité de l'énonciation: la fidélité théorique de $\mathrm{D}$. Evans à la révélation chrétienne", Recherches de science religieuse, t. 69, no 4 (1981), pp. 545-572; "Richard Rorty ou la boîte blanche de la communication ", Critique, t. XXXVII, no 417 (1982), p. 130-151; "L.e filtre de vérité. La jouissance des interlocuteurs et la croix des philosophes », Critique, t. XXXIX, no 430, pp. 215-231; «Cynisme ou pragmatisme? Le temps du jugement ", Critique, t. XLII, $\mathrm{n}^{\circ}$ 464-465 (1986); "Peut-on guérir de la politique?", Critique, t. XLIV, no 493-494 (1988), pp. 507-530; "Pragmatique et ontologie", dans l'Encyclopidie philosophique universelle, t. I, L'Univers philosophique, P.U.F., Paris, 1989, p. 512-520; "La sensibilisation de la raison 
dans l'anthropologie pragmatique ", dans Critique de la raison pragmatique. La transformation pragmatique, sous la direction de J. Poulain, Paris, Les Éditions du Cerf, 1991, pp. 187-204; “L'objectivité de l'objet ou l'enjeu philosophique de la sémantique ", Langages, $25^{\mathrm{e}}$ année, no 103 (1991), p. 66-82.

28. Cf. J. Poulain, "Le filtre de vérité. La jouissance des interlocuteurs et la croix des philosophes", p. 221.

29. Comme exemple, on peut donner le jugement de perception par opposition au jugement objectif, dans la CRP. Ce stade anté-prédicatif pourrait bien être celui de la synthèse apophantique, puisqu'on ne considère plus celle-ci comme un aboutissement.

30. F. Marty et A. Delamarre, dans E. Kant, Oeuvres philosophiques, vol. 1, p. 1591, note 1 de la p. 817.

31. Ak. III, 79; A 57 B 82; CRP, p. 80.

32. Über Gewissheit, § 124.

33. T 5.5151.

34. J. Poulain, "Cynisme ou pragmatisme »? Le temps du jugement ", p. 78. Souligné ajouté.

35. «Tout jugement, du seul fait qu'il arrive et qu'il se reconnaisse aussi vrai qu'il a dû se penser vrai pour pouvoir être pensé, est déjà aussi efficace qu'il peut l'être et que peut le rendre le consensus ». (J. Poulain, dans "Le temps du jugement», p. 78.)

36. "Peut-on guérir de la politique?», p. 511.

37. Ibid., p. 530.

38. La Critique de la faculté de juger et l'Anthropologie du point de vue pragmatique décrivent le jugement comme la faculté permettant de déterminer si une éventualité est ou non un cas relevant d'une règle, ce qui fait du jugement un acte individuel, pour lequel il ne saurait à proprement parler y avoir de préceptes. Cf. Anthropologie du point de vue pragmatique, dans CEuvres philosophiques, $t$. III, Paris, Gallimard, 1986, $\S 42$. Wittgenstein aurait sans doute préféré qu'on I'identifie à cette conception du jugement plutôt qu'à celle de la Critique de la raison pure. C. Stetter, dans "Kant und Wittgenstein " (Revue internationale de philosophie, vol. 45, $\mathrm{n}^{\circ} 176$ (1991), pp. 5975), associe le Tractatus à la seconde partie de la Critique de la faculté de juger, et les Investigations à la première partie. Il resterait à voir comment les deux conceptions du jugement qui sont présentées dans la première et la dernière Critiques s'articulent l'une à l'autre. La comparaison Kant/Wittgenstein peut nous mettre sur la voie. La maxime du "penser par soi-même " qui a été attribuée à la $C R P$ est également attribuable à Wittgenstein, si on étudie les quelques autres emplois du terme " jugement » dans les PU. Comment Kant (si on le réduit à la $C R P$ ) et Wittgenstein, en partant d'une définition semblable, peuvent-ils aboutir à des positions opposées 
(Kant étant subjectiviste là où Wittgenstein fait partie du grand courant suprasubjectif)? Un début de réponse pourrait être que si W'ttgenstein (comme Kant dans la CFJ et dans l'Anthropologie) considère le jugement comme un acte individuel et imprévisible, il pourrait aussi le décrire comme faisant partie des actes qu'un homme ne saurait faire une seule fois dans sa vie, comme il le dit déjà de ce qu'on appelle "suivre une règle » (PU 199). En d'autres, termes, le jugement est une "pratique» pour Wittgenstein. Et comme cette pratique " aveugle " ne suffit pas à rendre au jugement sa néccssité et son objectivité, on en vient à concevoir l'urgence d'un retour au primat de la raison théorique. 